BMJ

Open

Gastroenterology

\section{Persistent constipation and abdominal adverse events with newer treatments for constipation}

To cite: Sonu I,

Triadafilopoulos G,

Gardner JD. Persistent constipation and abdominal adverse events with newer treatments for constipation. BMJ Open Gastro 2016;3: e000094. doi:10.1136/ bmjgast-2016-000094

- Additional material is published online only. To view please visit the journal online (http://dx.doi.org/10. 1136/bmjgast-2016-000094)

Received 5 April 2016 Revised 3 May 2016 Accepted 6 May 2016
${ }^{1}$ Stanford University School of Medicine, Palo Alto, California, USA

${ }^{2}$ Science for Organizations, Inc., Mill Valley, California, USA

Correspondence to Dr Irene Sonu; isonu@stanford.edu

\begin{abstract}
Background: Clinical trials of several new treatments for opioid-induced constipation (OIC), chronic idiopathic constipation (CIC) and constipationpredominant irritable bowel syndrome (IBS-C) have focused on differences between subjects relieved of constipation with placebo and active treatment. Patients and clinicians however, are more interested in the probability these treatments provide actual relief of constipation and its associated symptoms.

Methods: We searched the medical literature using MEDLINE and Cochrane central register of controlled trials. Randomised, placebo-controlled trials that examined the use of methylnaltrexone, naloxegol, lubiprostone, prucalopride or linaclotide in adults with OIC, CIC and IBS-C were eligible for inclusion. The primary efficacy measure was relief of constipation. Adverse event data for abdominal symptoms were also analysed.
\end{abstract}

Key results and findings: 25 publications were included in our analyses. The proportion of constipated individuals with active treatment was significantly lower than the proportion with placebo; however, in 15 of these 20 trials analysed, a majority of patients remained constipated with active treatment. Analyses of adverse event data revealed that the percentage of participants who experienced abdominal pain, diarrhoea and flatulence with active treatment was higher than that with placebo in the majority of trials analysed.

Conclusions: Newer pharmacological treatments for constipation are superior to placebo in relieving constipation, but many patients receiving active treatment may remain constipated. In addition, all 5 of the treatments studied are accompanied by no change or a possible increase in the prevalence of abdominal symptoms, such as abdominal pain, diarrhoea and flatulence.

\section{INTRODUCTION}

Constipation affects up to $27 \%$ of the US population, and its incidence has been increasing. ${ }^{1}$ Chronic constipation imposes a high burden of healthcare costs in that it results in over two million physician visits and nearly 100000 hospitalisations per year. $^{2} \quad 3 \quad$ Further, constipation-related

\section{Summary box}

What is already known about this subject?

- Chronic constipation is a common problem that is difficult to treat.

- Patients often have continued symptoms despite conventional therapies.

- Newer pharmacological agents are available for patients with continued symptoms despite conventional therapies.

What are the new findings?

- In most clinical trials, a majority of patients treated with a newer agent remained constipated.

- These findings were seen across three types of constipation.

- These newer agents also do not change or actually increase the percentage of individuals with symptoms such as abdominal pain, diarrhoea and flatulence.

How might it impact on clinical practice in the foreseeable future?

- Clinicians should anticipate a high probability that with one of the newer treatments for constipation, a patient will remain constipated with persistent abdominal symptoms.

emergency department visits have increased by $42 \%$ from 2006 to 2011 , resulting in a $121 \%$ increase in associated costs. ${ }^{4}$ Chronic constipation impairs the quality of life and can result in increased psychological stress and depression. ${ }^{5}{ }^{6}$ It is also often accompanied by other symptoms, such as abdominal pain and discomfort, stomach cramping, bloating and gas pain. ${ }^{7}$

There are different difficult defaecation types of constipation. Chronic idiopathic constipation (CIC) is defined as constipation in individuals with no apparent physiological abnormality. ${ }^{8} \quad 9$ Constipation-predominant irritable bowel syndrome (IBS-C) is defined by the presence of recurrent abdominal pain or discomfort at least 3 days/month over a 3-month period, which is accompanied by at least two of the following: improvement with 
defaecation; onset associated with change of frequency of stool; or onset associated with change in form of stool. ${ }^{9}$ Opioid-induced constipation (OIC) is defined as constipation in individuals taking opioid medications. Up to $81 \%$ of patients taking opioids experience constipation, which can result in a significant impact on their quality of life and activities of daily living, ${ }^{10}$ and the incidence of OIC is expected to rise due to the increased number of narcotic prescriptions dispensed in the USA. Patients with OIC frequently have continued constipation despite the use of a laxative. ${ }^{11} 12$

Treatment of constipation initially involves fibre as well as over-the-counter osmotic and stimulant laxatives, ${ }^{13}$ but many patients continue to have symptoms. For such patients, the American Gastroenterological Association recommends treatment with one of the newer pharmacological agents that have been approved by the US Food and Drug Administration (FDA), or the European Medicines Agency (EMA)-lubiprostone (FDA approval 1, 2006), methylnaltrexone (FDA approval 4, 2008), prucalopride (EMA approval 10, 2009), linaclotide (FDA approval 8, 2012) and naloxegol (FDA approval 9, 2014). ${ }^{13}{ }^{14}$ In clinical trials, each treatment has been statistically significantly superior to placebo in relieving constipation. ${ }^{15-39}$ One problem, however, is that there is no clear, consistent relationship between the effect of a treatment on physiological processes in a constipated patient and its clinical effect on constipation.

The aim of our analyses was to examine the ability of these newer pharmacological agents to provide relief of constipation and its associated symptoms, including abdominal pain, diarrhoea, flatulence and bloating.

\section{METHODS}

We searched the medical literature using MEDLINE and the Cochrane central register of controlled trials, to identify publications that reported effects of these newer treatments on constipation. Search terms included the following as free-text terms: methylnaltrexone, naloxegol, lubiprostone, prucalopride or linaclotide. These terms were combined using the set operator AND with trials identified with the terms constipation, irritable bowel syndrome, opioid or trial. This search returned 1432 publications as of 1 March 2015. Some of these publications reported meta-analyses of effects of a particular treatment on constipation; ${ }^{40-44}$ however, we did not include these publications in our analyses because they did not contain data that enabled us to determine the proportion of individuals who remained constipated with active treatment or placebo.

From the initial search, we selected all publications of trials in adult individuals that were placebo-controlled and that reported data for relief of constipation as well as adverse event data $(\mathrm{N}=25)$. From these 25 publications, we selected all publications that reported the number or percentage of participants with relief of constipation $(\mathrm{N}=20)$. Of the original 25 publications, 5 reported average number of bowel movements during a given period of time, or the change from number of baseline bowel movements instead of percentage, or number of individuals with relief of constipation. ${ }^{21} 2426$

Data were extracted by two investigators (IS and JG) and placed onto a Microsoft Excel spreadsheet (Windows V.14.4.9, Microsoft, Redmond, Washington, USA). Each investigator created a separate database. The final 'locked database' was created after resolving all discrepancies between the two individual databases.

The primary efficacy measure was relief of constipation defined as (1) rescue-free laxation within 4 hours of first dose of study drug, (2) $\geq 3$ complete spontaneous bowel movements (CSBMs) per week, and $\geq 1$ CSBM per week from baseline for $75 \%$ of weeks, (3) $\geq 3-4$ spontaneous bowel movements per week during week 4 of treatment or (4) average of $\geq 3$ CSBMs per week over a 12-week period.

For each publication and for each treatment we calculated the percentage of patients who remained constipated at the end of the trial (ie, the percentage of individuals who failed to experience relief of constipation) as well as the percentage of participants who reported an abdominal symptom as an adverse eventabdominal pain, diarrhoea, flatulence or abdominal distension/bloating. In some instances, such as with abdominal pain and diarrhoea, the number of trials reporting values for one of these symptoms is greater than the number of trials included in our analyses of constipation because a trial may have reported adverse event data but did not report data that enabled us to calculate the percentages of individuals who remained constipated with active treatment and with placebo. In other instances, such as with flatulence or abdominal distension/bloating, the number of trials reporting values for one of these symptoms is less than the number of trials included in our analyses of constipation, because these symptoms were not reported in all trials included in our analyses of constipation. A database containing the extracted data used for all analyses in the present paper is provided in online supplementary table S1.

One-way analysis of variance (ANOVA) followed by Tukey-Kramer Multiple Comparisons Test and Unpaired t-test with Welch correction were performed using GraphPad InStat V.3.10 for Windows; Sample size was calculated using GraphPad StatMate V.2.00 for Windows, GraphPad Software, La Jolla California, USA. Figures were prepared using Microsoft Excel for Windows V.14.4.9, Microsoft, Redmond, Washington, USA.

\section{RESULTS}

Twenty trials included sufficient information to calculate the number and percentage of participants who remained constipated at the end of the trial. Constipation was an entry criterion for each trial. Figure 1 displays the percentage of individuals who received active treatment or placebo and were 
CONSTIPATION WITH CHRONIC IDIOPATHIC CONSTIPATION

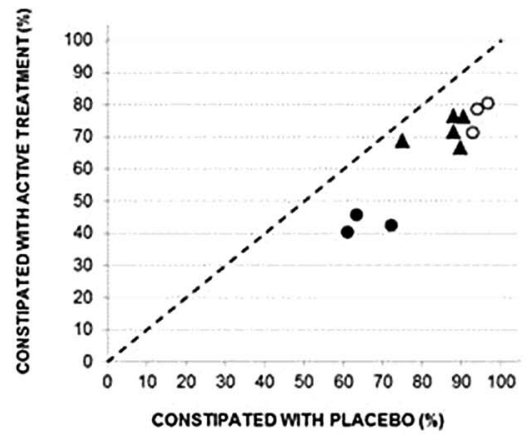

- Llubiprostone olinaclotide apRUCALOpride
CONSTIPATION WITH OPIOIDINDUCED CONSTIPATION

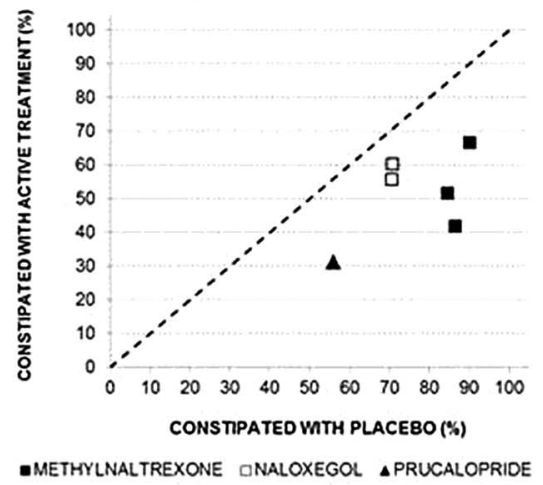

CONSTIPATION WITH IRRITABLE BOWEL SYNDROME-CONSTIPATION

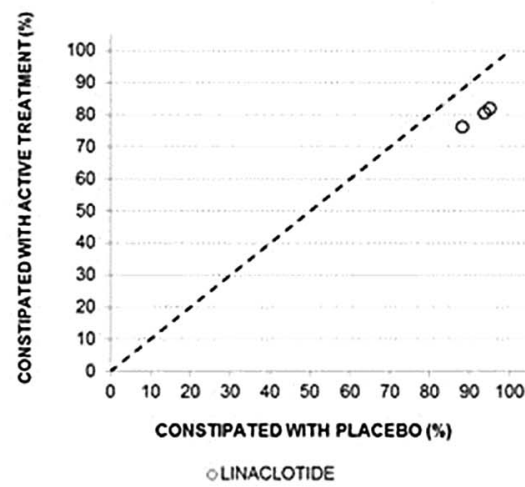

Figure 1 Relationship between constipation with active treatment and that with placebo. The percentage of individuals who were constipated with active treatment is plotted as a function of the corresponding value with placebo. If more than one dose of active treatment was tested in a particular trial, the value from the highest dose is analysed. The dashed diagonal line is the identity line. Symbols above the identity line indicate that the value for constipation with active treatment is higher than the corresponding value with placebo, and values below the identity line indicate the opposite.

constipated at the end of the trial stratified by type of constipation and then by active treatment. Results from each trial were below the identity line, indicating that the percentage of constipated patients with active treatment was less than the percentage of constipated individuals with placebo in the same trial. In all figures, the vertical and horizontal distances from any point to the identity line form the two equal sides of an isosceles triangle and represent the magnitude of the difference between active treatment and placebo.

Figure 1 (left) displays results from 11 trials in individuals with CIC. Results from the three trials of lubiprostone differed significantly from the five trials of prucalopride, and from the three trials of linaclotide (ANOVA $\mathrm{p}<0.0001$ for active treatment and $\mathrm{p}<0.0005$ for placebo). These results with placebo treatment indicate that with respect to the prevalence of constipation, participants in one group of trials are not exchangeable with participants in the other two groups of trials. In the lubiprostone trials, values for constipation with active treatment were significantly lower than corresponding values for active treatment in the prucalopride trials $(\mathrm{p}<0.0001$ Tukey-Kramer Multiple Comparisons Test) and in the linaclotide trials $(\mathrm{p}<0.0001$ Tukey-Kramer Multiple Comparisons Test). Also, in each of the eight trials of prucalopride and linaclotide, a majority of patients $(67-81 \%)$ remained constipated with active treatment, whereas in the lubiprostone trials, only $40-46 \%$ of participants remained constipated with active treatment. Also, in the lubiprostone trials, values for constipation with placebo treatment were significantly lower than corresponding values for active treatment in the prucalopride trials $\quad(\mathrm{p}<0.001 \quad$ Tukey-Kramer Multiple Comparisons Test) and in the linaclotide trials $(\mathrm{p}<0.0001$ Tukey-Kramer Multiple Comparisons Test). Moreover, these results raise the possibility that the increased effectiveness of lubiprostone compared with prucalopride and linaclotide in relieving idiopathic chronic constipation may have occurred simply because participants enrolled in the lubiprostone trials were less likely to remain constipated at the end of the trial regardless of whether they received placebo or active treatment. Evidence to support this possibility is that mean difference $(95 \%$ CI) between the percentages of individuals constipated with active treatment and placebo in the lubiprostone trials (23 (6-39)) was not significantly different from the differences in the prucalopride $(14(6-22))$ or linaclotide $(18(5-31))$ trials.

Figure 1 (middle) displays results from six trials in patients with OIC. Values for constipation with active treatment in the three methylnaltrexone trials were not significantly different from values with active treatment in the two naloxegol trials $(\mathrm{p}=0.602$; unpaired t-test with Welch correction). On the other hand, values for constipation with placebo treatment in the three methylnaltrexone trials were significantly higher than values with placebo treatment in the two naloxegol trials $(p=0.0099$; unpaired t-test with Welch correction). The single trial of prucalopride in OIC had a value for constipation with placebo treatment that was lower than values with placebo treatment in the methylnaltrexone and naloxegol trials making it likely that with respect to the prevalence of constipation, participants in trials with one of these three treatments are not exchangeable with individuals in trials with the other two treatments. In the two naloxegol trials, and two of the three methylnaltrexone trials, a majority of patients remained constipated with active treatment. In one of the methylnaltrexone trials, only $42 \%$ of participants remained constipated with active treatment; and in the single prucalopride trial, $31 \%$ of patients remained constipated with active treatment. Compared to the trials of methylnaltrexone and naloxegol, the increased effectiveness of the single trial of prucalopride in relieving OIC may have occurred 
simply because participants enrolled in the prucalopride trial were less likely to remain constipated at the end of the trial regardless of whether they received placebo or active treatment.

Figure 1 (right) illustrates results from three trials of linaclotide in IBS-C. Although values for constipation with active treatment were lower than corresponding values with placebo $(\mathrm{p}=0.0012$; paired $\mathrm{t}$-test $)$, the proportion of patients who remained constipated ranged from $88 \%$ to $95 \%$ with placebo, and from $76 \%$ to $82 \%$ with active treatment.

Considering all 20 trials with relief of constipation as an efficacy endpoint, as many as $44 \%$ of participants were no longer constipated after being treated with placebo. The percentage of individuals who remained constipated with active treatment varied from $31 \%$ to $82 \%$, and in 15 of the 20 trials, a majority of participants remained constipated with active treatment.

Twenty-five trials gave the number or percentage of participants who reported abdominal pain as a spontaneous adverse event. Figure 2 displays the percentage of participants who reported abdominal pain with active treatment, or placebo stratified by type of constipation, and then by active treatment. Results from 22 of the 25 trials were above the identity line, indicating that the percentage of patients with abdominal pain with active treatment was greater than the percentage of individuals with abdominal pain with placebo in the same trial.

Figure 2 (left) displays results for abdominal pain from 13 trials in participants with CIC. Results from the five trials of prucalopride differed significantly from the five trials of lubiprostone, and from the three trials of linaclotide (ANOVA $\mathrm{p}=0.0049$ for active treatment and $\mathrm{p}=0.0392$ for placebo). These results with placebo treatment indicate that with respect to the prevalence of abdominal pain, individuals in one group of trials are not exchangeable with participants in the other two groups of trials. In the prucalopride trials, values for abdominal pain with active treatment were significantly higher than corresponding values for active treatment in the lubiprostone trials $(\mathrm{p}<0.05$ Tukey-Kramer Multiple Comparisons Test) and in the linaclotide trials $(\mathrm{p}<0.05$ Tukey-Kramer Multiple Comparisons Test). In the prucalopride trials, values for abdominal pain with placebo were significantly higher than corresponding values in the lubiprostone trials $(\mathrm{p}<0.05$ Tukey-Kramer Multiple Comparisons Test) but not in the linaclotide trials ( $p>0.05$ Tukey-Kramer Multiple Comparisons Test). Overall, the percentage of patients who experienced abdominal pain with placebo varied from $0 \%$ to $19 \%$, and the percentage of individuals who experienced abdominal pain with active treatment varied from $0 \%$ to $38 \%$. The percentages of patients with abdominal pain with active treatment were higher than the corresponding percentage with placebo in five of the five prucalopride trials, four of the five lubiprostone trials, but in only one of the three linaclotide trials.

Figure 2 (middle) displays results for abdominal pain from eight trials in participants with OIC. In the three methylnaltrexone trials, values for abdominal pain with active treatment or with placebo were not significantly different from corresponding values in the three naloxegol trials (active treatment $\mathrm{p}=0.725$; placebo $\mathrm{p}=0.894$; unpaired t-test with Welch correction). Values from the single prucalopride trial were in the same range as values from the trials of methylnaltrexone and naloxegol, whereas values from the single lubiprostone trial were lower than values from the other seven trials. Overall, the percentage of patients who experienced abdominal pain with placebo varied from $4 \%$ to $13 \%$, and the percentage of individuals who experienced abdominal pain with active treatment varied from $4 \%$ to $38 \%$. The percentages of participants with abdominal pain with active treatment were higher than the
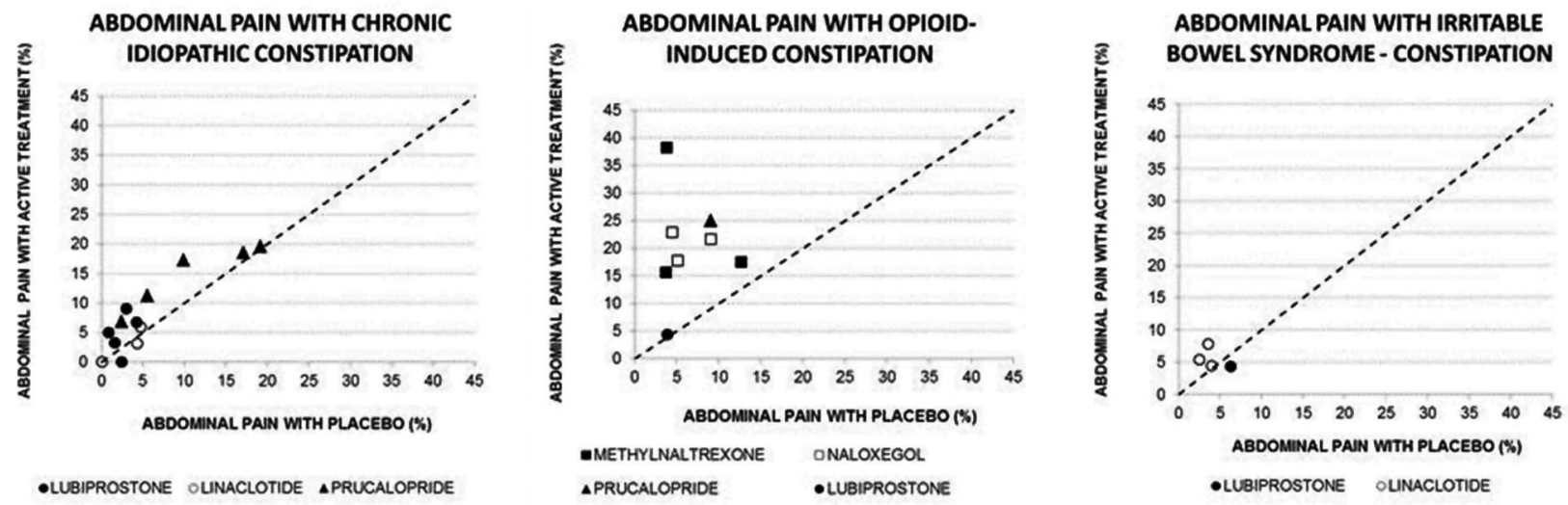

Figure 2 Relationship between abdominal pain with active treatment and that with placebo. The percentage of individuals with abdominal pain with active treatment is plotted as a function of the corresponding value with placebo. If more than one dose of active treatment was tested in a particular trial, the value from the highest dose is analysed. The dashed diagonal line is the identity line. Symbols above the identity line indicate that the value for abdominal pain with active treatment is higher than the corresponding value with placebo, and values below the identity line indicate the opposite. 
corresponding percentages with placebo in each of the eight trials of treatments for OIC.

Figure 2 (right) illustrates results for abdominal pain from three trials of linaclotide and one trial of lubiprostone in patients with IBS-C. Values for abdominal pain with active treatment were higher than corresponding values with placebo in each of the linaclotide trials but not in the lubiprostone trial.

Considering all 25 trials with abdominal pain reported as an adverse event, $0-19 \%$ of participants reported abdominal pain with placebo, and $0-38 \%$ of individuals reported abdominal pain with active treatment. Figure 2 also illustrates that with placebo, there were no significant differences among results from different types of constipation with respect to values for percentages of patients with abdominal pain (mean CIC 5.8\%; OIC $6.5 \%$; IBS-C $4.1 \%$; ANOVA $\mathrm{p}=0.724)$. In contrast, with active treatment, values for percentages of subjects with abdominal pain and OIC (mean 20.3\%) were significantly higher (ANOVA $\mathrm{p}=0.0019$ ) than those for CIC (mean 8.2\%; $\quad \mathrm{p}<0.01 \quad$ Tukey-Kramer Multiple Comparisons Test), and those for IBS-C (5.5\%; $\mathrm{p}<0.01$ Tukey-Kramer Multiple Comparisons Test). Results with active treatment for abdominal pain with CIC were not significantly different from those for IBS-C $\quad(p>0.05$ Tukey-Kramer Multiple Comparisons Test).

Twenty-five trials gave the number or percentage of patients who reported diarrhoea as a spontaneous adverse event. Figure 3 displays the percentage of patients who reported diarrhoea with active treatment, or placebo stratified by type of constipation and then by active treatment. Results from 24 of the 25 trials were above the identity line, indicating that the percentage of individuals with diarrhoea with active treatment was greater than the percentage of patients with diarrhoea with placebo in the same trial.
Figure 3 (left) displays results for diarrhoea from 13 trials in participants with CIC. There were no significant differences among the five trials of prucalopride, the five trials of lubiprostone and the three trials of linaclotide for the percentages of patients with diarrhoea with active treatment or placebo (ANOVA $\mathrm{p}=0.683$ for active treatment and $\mathrm{p}=0.054$ for placebo). Overall, the percentage of individuals who experienced diarrhoea with placebo varied from $0 \%$ to $8 \%$, and the percentage of participants who experienced diarrhoea with active treatment varied from $3 \%$ to $22 \%$. The percentages of patients with diarrhoea with active treatment were higher than the corresponding percentage with placebo in all trials of prucalopride, lubiprostone and linaclotide.

Figure 3 (middle) displays results for diarrhoea from seven trials in participants with OIC. In the two methylnaltrexone trials, values for diarrhoea with active treatment or with placebo were not significantly different from corresponding values in the three naloxegol trials (active treatment $\mathrm{p}=0.075$; placebo $\mathrm{p}=0.293$; unpaired t-test with Welch correction). Values from the single prucalopride trial, and from the single lubiprostone trial were in the same range as values from the trials of methylnaltrexone and naloxegol. Overall, the percentage of participants who experienced diarrhoea with placebo varied from $2 \%$ to $6 \%$, and the percentage of individuals who experienced diarrhoea with active treatment varied from $6 \%$ to $13 \%$. The percentages of patients with diarrhoea with active treatment were higher than the corresponding percentages with placebo in each of the seven trials of treatments for OIC.

Figure 3 (right) illustrates results for diarrhoea from three trials of linaclotide and two trials of lubiprostone in IBS-C. In the two lubiprostone trials, values for
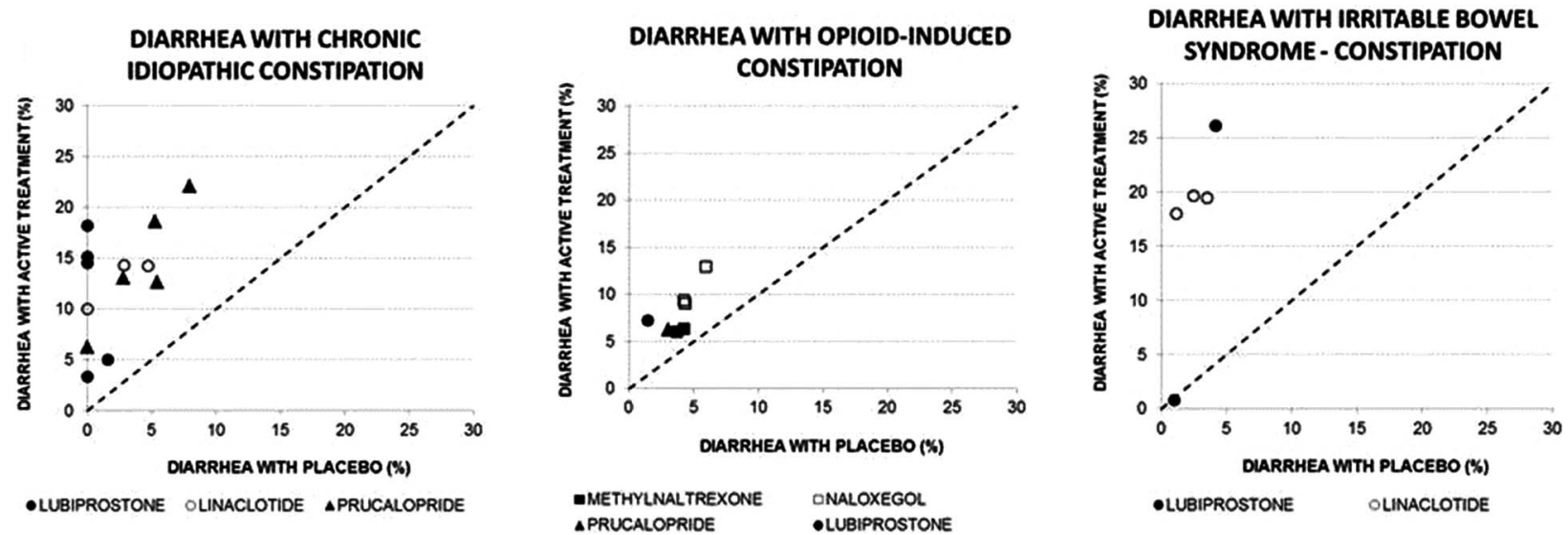

Figure 3 Relationship between diarrhoea with active treatment and that with placebo. The percentage of participants with diarrhoea with active treatment is plotted as a function of the corresponding value with placebo. If more than one dose of active treatment was tested in a particular trial, the value from the highest dose is analysed. The dashed diagonal line is the identity line. Symbols above the identity line indicate that the value for diarrhoea with active treatment is higher than the corresponding value with placebo, and values below the identity line indicate the opposite. 
diarrhoea with active treatment or with placebo were not significantly different from corresponding values in the three linaclotide trials (active treatment $\mathrm{p}=0.734$; placebo $\mathrm{p}=0.926$; unpaired t-test with Welch correction). Overall, the percentage of individuals with IBS-C who experienced diarrhoea with placebo varied from $1 \%$ to $4 \%$, and the percentage of patients who experienced diarrhoea with active treatment varied from $1 \%$ to $26 \%$. The percentages of participants with diarrhoea with active treatment were higher than the corresponding percentages with placebo in each of the three linaclotide trials and one of the two lubiprostone trials.

Considering all 25 trials with diarrhoea as adverse events, $0-8 \%$ of participants reported diarrhoea with placebo, and $1-26 \%$ of individuals reported diarrhoea with active treatment. Figure 3 also illustrates that with placebo, there were no significant differences among results from different types of constipation with respect to values for percentages of patients with diarrhoea (mean CIC 3.8\%; OIC 2.4\%; IBS-C 2.5\%; ANOVA $\mathrm{p}=0.356)$. Similarly, with active treatment, there were no significant differences among results from different types of constipation with respect to values for percentages of individuals with diarrhoea (mean CIC 12.9\%; OIC $8.2 \%$; IBS-C $16.8 \%$; ANOVA $p=0.056$ ). On the other hand, the $p$ value of 0.056 raises the possibility that with more trials, there might be significant differences among the active treatments with respect to values for percentages of patients with diarrhoea.

Seventeen trials gave the number or percentage of individuals who reported flatulence as a spontaneous adverse event. Figure 4 displays the percentage of participants who reported flatulence with active treatment or placebo stratified by type of constipation and then by active treatment. Percentage of patients with flatulence with active treatment was greater than the percentage of individuals with flatulence with placebo in the same trial.

Figure 4 (left) displays results for flatulence from eight trials in patients with CIC. There were no significant differences among two trials of prucalopride, the three trials of lubiprostone and the three trials of linaclotide for the percentages of individuals with flatulence with active treatment or placebo (ANOVA $\mathrm{p}=0.187$ for active treatment, and $\mathrm{p}=0.185$ for placebo). Overall, the percentage of patients who experienced flatulence with placebo varied from $0 \%$ to $9 \%$, and the percentage of participants who experienced flatulence with active treatment varied from $0 \%$ to $8 \%$. The percentages of patients with flatulence with active treatment were higher than the corresponding percentage with placebo in one of two trials of prucalopride, two of three trials of lubiprostone, and none of the three trials of linaclotide.

Figure 4 (middle) displays results for flatulence from six trials in patients with OIC. In the two methylnaltrexone trials, values for flatulence with active treatment or with placebo were not significantly different from corresponding values in the three naloxegol trials (active treatment $\mathrm{p}=0.083$; placebo $\mathrm{p}=0.294$; unpaired $\mathrm{t}$-test with Welch correction). The value for flatulence with placebo from the single lubiprostone trial was in the same range as values with placebo from the trials of methylnaltrexone and naloxegol, but the value for flatulence with lubiprostone was lower than values with methylnaltrexone and naloxegol. Overall, the percentage of individuals who experienced flatulence with placebo varied from $1 \%$ to $7 \%$, and the percentage of participants who experienced flatulence with active treatment varied from $4 \%$ to $14 \%$. The percentages of patients with flatulence with active treatment were higher than the corresponding percentages with placebo in each of the six trials of treatments for OIC.
FLATULENCE WITH CHRONIC IDIOPATHIC CONSTIPATION

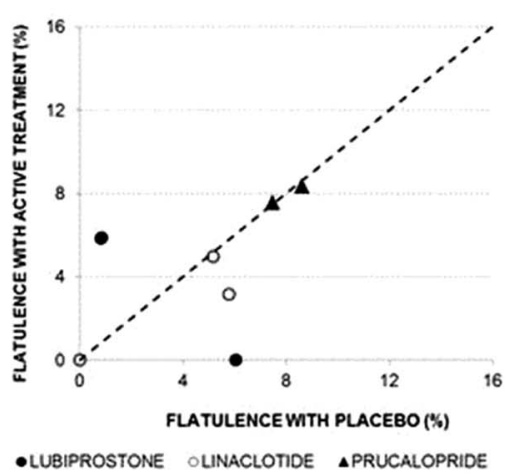

FLATULENCE WITH OPIOID-INDUCED CONSTIPATION

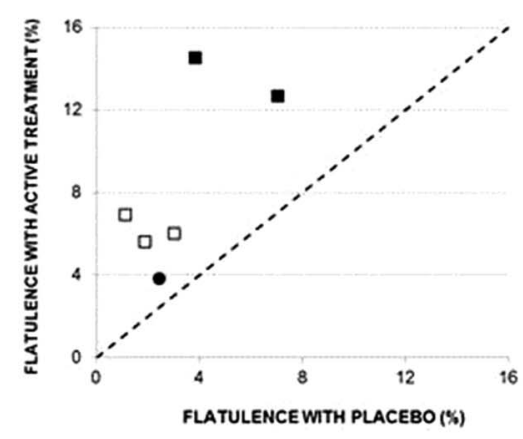

- METHYLNALTREXONE INALOXEGOL •lUBIPROSTONE
FLATULENCE WITH IRRITABLE BOWEL SYNDROME - CONSTIPATION

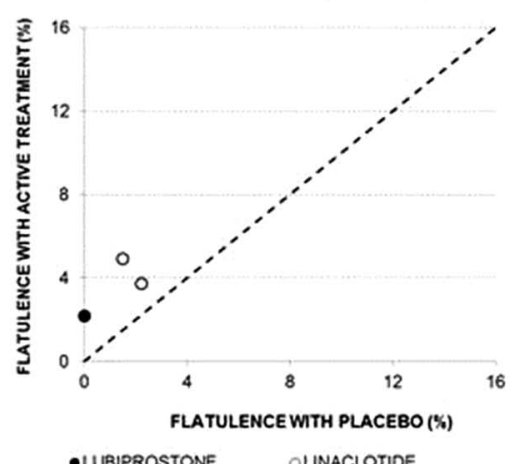

- lubiprostone olinaclotioe

Figure 4 Relationship between flatulence with active treatment and that with placebo. The percentage of patients with diarrhoea with active treatment is plotted as a function of the corresponding value with placebo. If more than one dose of active treatment was tested in a particular trial, the value from the highest dose is analysed. The dashed diagonal line is the identity line. Symbols above the identity line indicate that the value for flatulence with active treatment is higher than the corresponding value with placebo, and values below the identity line indicate the opposite. 
Figure 4 (right) illustrates results for flatulence from two trials of linaclotide and one trial of lubiprostone in IBS-C. Overall, the percentage of participants with IBS-C who experienced flatulence with placebo varied from $0 \%$ to $2 \%$, and the percentage of individuals who experienced flatulence with active treatment varied from $2 \%$ to $5 \%$. The percentages of patients with flatulence with active treatment were higher than the corresponding percentages with placebo in each of the three trials of treatments for IBS-C.

Considering all 17 trials with flatulence as adverse events, $0-9 \%$ of participants reported flatulence with placebo and $0-14 \%$ of patients reported flatulence with active treatment. Figure 4 also illustrates that with placebo, there were no significant differences among results from different types of constipation with respect to values for percentages of individuals with flatulence (mean CIC 4.4\%; OIC 3.2\%; IBS-C 1.2\%; ANOVA p=0.262). Similarly, with active treatment, there were no significant differences among results from different types of constipation with respect to values for percentages of patients with flatulence (mean CIC 4.5\%; OIC 8.2\%; IBS-C 3.6\%; ANOVA $\mathrm{p}=0.102)$.

Ten trials gave the number or percentage of participants who reported bloating or abdominal distension as a spontaneous adverse event. Figure 5 displays the percentage of individuals who reported bloating or abdominal distension with active treatment or placebo stratified by type of constipation and then by active treatment. Results from five of the 10 trials were above the identity line, indicating that the percentage of patients with bloating or abdominal distension with active treatment was greater than the percentage of participants with bloating or abdominal distension with placebo in the same trial.

Considering all 10 trials with bloating or abdominal distension as spontaneous adverse events, the percentage of patients who experienced flatulence with placebo varied from $1 \%$ to $10 \%$, and the percentage of participants who experienced flatulence with active treatment varied from $0 \%$ to $11 \%$. Furthermore, of these 10 trials, seven compared lubiprostone with placebo. In the three trials of CIC that tested lubiprostone (figure 5 (left)), values for bloating or abdominal distension with lubiprostone were lower than corresponding values with placebo. In the single trial of OIC that tested lubiprostone (figure 5 (middle) ), the value for bloating or abdominal distension with lubiprostone was higher than the corresponding value with placebo. In the two trials of IBS-C that tested lubiprostone (figure 5 (right)), values for bloating or abdominal distension with lubiprostone were lower than corresponding values with placebo in one of the two trials. In the two trials of IBS-C that tested linaclotide (figure 5 (right)), values for bloating or abdominal distension with linaclotide were higher than corresponding values with placebo in both trials.

\section{DISCUSSION}

In the trials of treatment for constipation, all participants had to be constipated at study entry. We were surprised to find, however, that although the percentage of patients who remained constipated at the end of the trial with active treatment was lower than that with placebo in all 20 trials, in 15 of $20(75 \%)$ trials, a majority of subjects who received active treatment remained constipated at the end of the trial. This phenomenon occurred with treatment for CIC, OIC and IBS-C. In those trials where only a minority of remained constipated with active treatment, a minority of participants also tended to remain constipated with placebo. Thus, this apparent increased effectiveness of active treatment may have occurred simply because participants enrolled in the trials were less likely to remain constipated at the end of the trial, regardless of whether they received placebo or active treatment.
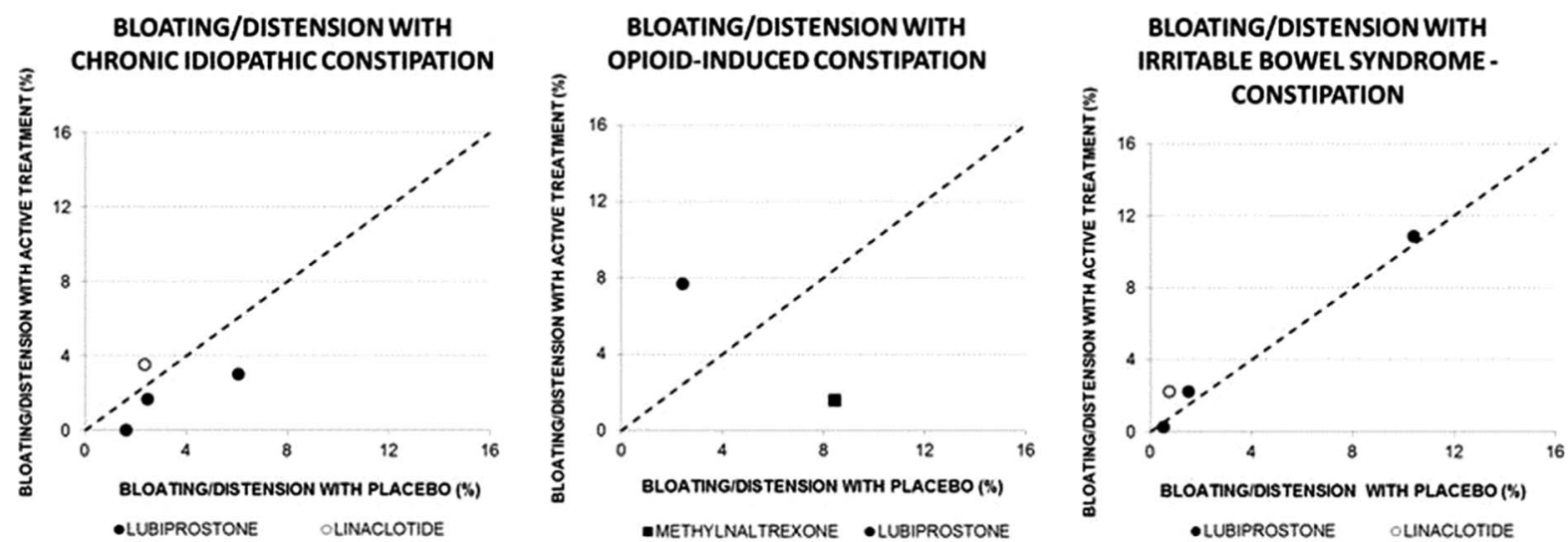

Figure 5 Relationship between bloating or distension with active treatment and that with placebo. The percentage of patients with diarrhoea with active treatment is plotted as a function of the corresponding value with placebo. If more than one dose of active treatment was tested in a particular trial, the value from the highest dose is analysed. The dashed diagonal line is the identity line. Symbols above the identity line indicate that the value for bloating or distension with active treatment is higher than the corresponding value with placebo, and values below the identity line indicate the opposite. 
Furthermore, across all trials, the percentage of individuals with an abdominal adverse effect was generally higher with active treatment than with the corresponding placebo. Thus, in a majority of trials, not only did most subjects remain constipated with active treatment, and also the prevalence of abdominal symptoms, such as abdominal pain, diarrhoea, and flatulence increased with active treatment compared with placebo. These side effects may have a profound impact on the patient, especially in pain-predominant syndromes like IBS-C, where treatment is not only focused on constipation relief and also in relieving pain, oftentimes with antidepressants and psychological interventions.

In the 20 trials of treatments for constipation, the percentage of individuals who received placebo and remained constipated at the end of the trial ranged from $56 \%$ to $97 \%$. In trials of CIC as well as of OIC, the percentage of patients who remained constipated in the placebo arms of the various active treatments differed significantly from each other. These findings indicate, for example, that in trials of CIC, subjects who received lubiprostone, prucalopride or linaclotide were not exchangeable with subjects in the other two groups. This variation makes it difficult for clinicians to decide on a particular treatment for constipation. This variation can also pose problems for investigators who design trials for treatments of constipation in that the number of subjects who will be required to detect a treatment effect of a particular magnitude will depend on the percentage of participants who are constipated with placebo treatment. For example, if $90 \%$ of patients who receive placebo will remain constipated at the end of the trial, a sample size of 60 patients per treatment group will have $90 \%$ power to detect a decrease of $24 \%$ with active treatment with a two-sided $\alpha$ of $0.05 .{ }^{45}$ On the other hand, if $60 \%$ of subjects who receive placebo will remain constipated at the end of the trial, a sample size of 90 subjects per treatment group will be required to have $90 \%$ power to detect a decrease of $24 \%$ with active treatment with a two-sided $\alpha$ of 0.05 .

The percentages of individuals with OIC who received active treatment and experienced abdominal pain were significantly higher than the percentages with CIC or IBS-C who received active treatment and experienced abdominal pain. This difference cannot be accounted for by the opioid receptor antagonist exerting a central effect and unblocking the analgesic effect of opioids, because possible changes in opioid-induced analgesia were examined in the clinical trials and none were found. ${ }^{15-19}$ An alternative possibility is that opioid-induced decreased bowel motility, which is believed to account for the constipation, is reversed by active treatment and the resulting increased bowel activity may produce abdominal pain.

A number of publications that we analysed mentioned the use of rescue medication, which refers to the unblinded use of treatment for constipation in addition to the blinded treatments under investigation. Although we believe that all trials probably employed rescue medication, some publications provided detailed descriptions of the conditions under which rescue medication was to be used, such as three consecutive days without a bowel movement, ${ }^{15}$ 18-21 26 28-31 333739 whereas, other publications only mentioned use of rescue medication in passing $^{34} 38$ or not at all. Except for the reports of trials with methylnaltrexone, which used an efficacy endpoint of laxation within 4 hours of administration of treatment, no publication provided a clear description of how administration of rescue medication influenced analysis of the trial results. Except for the trials of methylnaltrexone, all other trials defined constipation as fewer than three bowel movements during a 7-day period, and it seems to us that the use of rescue medication may have inflated the proportion of patients who experienced constipation in a given trial that did not involve methylnaltrexone.

Each trial included in our analyses assessed adverse events using the standard open-ended or free-response format. This has the advantage of not depending on asking individuals about symptoms that have been prespecified by the investigator or by a standard questionnaire, and therefore, may generate findings that might otherwise have been overlooked-a particularly important issue for pharmaceutical products. Also, an openended response is likely to reduce the 'noise' from positive responses from placebo participants, and thereby make it easier to detect adverse events associated with active treatments. A limitation of open-ended questions, however, is that it is not known whether a patient's failure to report a symptom occurred because there was no symptom or simply because it was not asked for.

Analyses of symptom data from the same trials using open-ended responses recorded in the adverse data compared to responses to prespecified questions regarding the same symptoms illustrate how these two approaches to symptom assessment can result in markedly different results. In our analyses, we included results from two trials of the effect of linaclotide in patients with CIC reported by Lembo et al. ${ }^{34}$ Combined results from these two trials indicated that with placebo, linaclotide $145 \mu \mathrm{g}$ and linaclotide $290 \mu \mathrm{g}$, the percentages of individuals with abdominal pain as an adverse event were $4.7 \%, 7.0 \%$ and $5.9 \%$, respectively; and the percentages of participants with abdominal bloating as an adverse event were $2.4 \%, 3.5 \%$ and $3.6 \%$, respectively. Chang et $a l^{46}$ analysed data from daily responses prespecified questions regarding abdominal symptoms in the same two trials, and reported that the percentages of participants who experienced abdominal pain, abdominal discomfort or abdominal bloating on at least 1 day during a 2-week baseline period were $91 \%, 96 \%$ and $97 \%$, respectively. We believe that the results from the analyses by Chang et al may represent a situation where a prespecified question increases the 'noise' in the data and obscures differences between placebo and active treatment. 
One limitation to our analyses is that grouping publications by the different clinical types of constipation, and then subdividing each group on the basis of the active treatment tested, we often ended up with a small number of trials for a given active treatment that may have prevented us from identifying differences among various treatments or their accompanying placebos. For example, for participants who reported abdominal bloating or distension, there were four trials of patients with CIC (three with lubiprostone and one with linaclotide), two trials of patients with OIC (one with lubiprostone and one with methylnaltrexone) and four trials of IBS-C (three with lubiprostone and one with linaclotide).

There was no consistent relationship among the percentages of individuals who experienced a particular abdominal symptom with active treatment. For example, there were significant differences among participants treated with lubiprostone, prucalopride or linaclotide with respect to the percentages of individuals with abdominal pain, but not with respect to the percentages of patients with diarrhoea or flatulence. We have also been unable to identify any particular pharmacological mechanism of action that might account for our finding increased abdominal symptoms with active treatment. Both linaclotide and lubiprostone stimulate intestinal secretion of fluid and electrolytes, ${ }^{47} 48$ and this action might account for diarrhoea; however, the values for the percentage of individuals who experienced diarrhoea with these agents were in the same range as those with prucalopride, which has a different mechanism of action. ${ }^{49}$ Further, there are few studies investigating the effect these medications have on gastrointestinal transit, and whether increasing transit has a direct effect on symptom improvement or the development of side effects.

In summary, although the newer pharmacological treatments for constipation are superior to placebo in relieving constipation, many patients receiving active treatment are left constipated. Further, all five of these new treatments are associated by either no change or possibly an increase in abdominal pain, diarrhoea and flatulence.

Contributors IS was responsible for the acquisition of data, analysis and interpretation of the data, drafting of the manuscript, critical revision of the manuscript for important intellectual content. GT was responsible for the study concept and design, analysis and interpretation of the data, critical revision of the manuscript for important intellectual content. JDG was responsible for study concept and design, acquisition of data, statistical analysis, analysis and interpretation of the data, drafting of the manuscript, critical revision of the manuscript for important intellectual content.

Funding Work by JDG was supported by Science for Organizations, Inc. Competing interests None declared.

Provenance and peer review Not commissioned; externally peer reviewed.

Data sharing statement Any unpublished data from the study is only available to the authors of the manuscript.

Open Access This is an Open Access article distributed in accordance with the Creative Commons Attribution Non Commercial (CC BY-NC 4.0) license, which permits others to distribute, remix, adapt, build upon this work non- commercially, and license their derivative works on different terms, provided the original work is properly cited and the use is non-commercial. See: http:// creativecommons.org/licenses/by-nc/4.0/

\section{REFERENCES}

1. Higgins PDR, Johanson JF. Epidemiology of constipation in North America: a systematic review. Am J Gastroenterol 2004;99:750-9.

2. Sonnenberg A, Koch TR. Physician visits in the United States for constipation: 1958 to 1986. Dig Dis Sci 1989;34:606-11.

3. Cai Q, Buono JL, Spalding WM, et al. Healthcare costs among patients with chronic constipation: a retrospective claims analysis in a commercially insured population. J Med Econ 2014;17:148-58.

4. Sommers T, Corban C, Sengupta N, et al. Emergency Department Burden of Constipation in the United States from 2006 to 2011. Am J Gastroenterol 2015;110:572-9.

5. Wald A, Hinds JP, Caruana BJ. Psychological and physiological characteristics of patients with severe idiopathic constipation. Gastroenterology 1989;97:932-7. http://www.ncbi.nlm.nih.gov/ pubmed/2777045

6. Wald A, Scarpignato C, Kamm MA, et al. The burden of constipation on quality of life: results of a multinational survey. Aliment Pharmacol Ther 2007;26:227-36.

7. Heidelbaugh JJ, Stelwagon M, Miller SA, et al. The spectrum of constipation-predominant irritable bowel syndrome and chronic idiopathic constipation: US survey assessing symptoms, care seeking, and disease burden. Am J Gastroenterol 2015;110:580-7.

8. Stewart WF, Liberman JN, Sandler RS, et al. Epidemiology of constipation (EPOC) study in the United States: relation of clinical subtypes to sociodemographic features. Am J Gastroenterol 1999;94:3530-40.

9. Longstreth GF, Thompson WG, Chey WD, et al. Functional bowel disorders. Gastroenterology 2006;130:1480-91.

10. Bell TJ, Panchal SJ, Miaskowski C, et al. The prevalence, severity, and impact of opioid-induced bowel dysfunction: results of a US and European patient survey (PROBE 1). Pain Med 2009;10:35-42.

11. Abramowitz L, Béziaud N, Labreze L, et al. Prevalence and impact of constipation and bowel dysfunction induced by strong opioids: a cross-sectional survey of 520 patients with cancer pain: DYONISOS study. J Med Econ 2013;16:1423-33.

12. Coyne KS, LoCasale RJ, Datto CJ, et al. Opioid-induced constipation in patients with chronic noncancer pain in the USA, Canada, Germany, and the UK: descriptive analysis of baseline patient-reported outcomes and retrospective chart review. Clinicoecon Outcomes Res 2014;6:269-81.

13. Bharucha AE, Dorn SD, Lembo A, et al. American gastroenterological association medical position statement on constipation. Gastroenterology 2013;144:211-17.

14. Brandt LJ. An evidence-based systematic review on the management of irritable bowel syndrome. Am J Gastroenterol 2009;104:S1-35.

15. Thomas J, Karver S, Cooney GA, et al. Methylnaltrexone for opioid-induced constipation in advanced illness. N Engl J Med 2008;358:2332-43.

16. Slatkin N, Thomas J, Lipman AG, et al. Methylnaltrexone for treatment of opioid-induced constipation in advanced illness patients. J Support Oncol 2009;7:39-46 (cited 2 Mar 2015). http:// www.ncbi.nlm.nih.gov/pubmed/19278178

17. Michna E, Blonsky ER, Schulman S, et al. Subcutaneous methylnaltrexone for treatment of opioid-induced constipation in patients with chronic, nonmalignant pain: a randomized controlled study. J Pain 2011;12:554-62.

18. Chey WD, Webster L, Sostek M, et al. Naloxegol for opioid-induced constipation in patients with noncancer pain. $N$ Engl J Med 2014;370:2387-96.

19. Webster L, Chey WD, Tack J, et al. Randomised clinical trial: the long-term safety and tolerability of naloxegol in patients with pain and opioid-induced constipation. Aliment Pharmacol Ther 2014;771-9.

20. Johanson JF, Morton D, Geenen J, et al. Multicenter, 4-week, double-blind, randomized, placebo-controlled trial of lubiprostone, a locally-acting type-2 chloride channel activator, in patients with chronic constipation. Am J Gastroenterol 2008;103:170-7.

21. Johanson JF, Ueno R. Lubiprostone, a locally acting chloride channel activator, in adult patients with chronic constipation: a double-blind, placebo-controlled, dose-ranging study to evaluate efficacy and safety. Aliment Pharmacol Ther 2007;25:1351-61.

22. Cryer B, Katz S, Vallejo R, et al. A randomized study of lubiprostone for opioid-induced constipation in patients with chronic noncancer pain. Pain Med 2014;15:1825-34. 
23. Barish CF, Drossman D, Johanson JF, et al. Efficacy and safety of lubiprostone in patients with chronic constipation. Dig Dis Sci 2010;55:1090-7.

24. Fukudo $\mathrm{S}$, Hongo $\mathrm{M}$, Kaneko $\mathrm{H}$, et al. Efficacy and safety of oral lubiprostone in constipated patients with or without irritable bowel syndrome: a randomized, placebo-controlled and dose-finding study. Neurogastroenterol Motil 2011;23:544-e205.

25. Fukudo S, Hongo M, Kaneko $\mathrm{H}$, et al. Lubiprostone increases spontaneous bowel movement frequency and quality of life in patients with chronic idiopathic constipation. Clin Gastroenterol Hepatol 2015;13:294-301.e5.

26. Johanson JF, Drossman DA, Panas R, et al. Clinical trial: phase 2 study of lubiprostone for irritable bowel syndrome with constipation. Aliment Pharmacol Ther 2008;27:685-96.

27. Drossman DA, Chey WD, Johanson JF, et al. Clinical trial: lubiprostone in patients with constipation-associated irritable bowe syndrome-results of two randomized, placebo-controlled studies. Aliment Pharmacol Ther 2009;29:329-41.

28. Camilleri M, Kerstens R, Rykx A, et al. A placebo-controlled trial of prucalopride for severe chronic constipation. $N$ Engl J Med 2008;358:2344-54

29. Quigley EMM, Vandeplassche L, Kerstens R, et al. Clinical trial: the efficacy, impact on quality of life, and safety and tolerability of prucalopride in severe chronic constipation-a 12-week, randomized, double-blind, placebo-controlled study. Aliment Pharmacol Ther 2009;29:315-28.

30. Tack J, van Outryve M, Beyens G, et al. Prucalopride (Resolor) in the treatment of severe chronic constipation in patients dissatisfied with laxatives. Gut 2009;58:357-65

31. Müller-Lissner S, Rykx A, Kerstens R, et al. A double-blind placebo-controlled study of prucalopride in elderly patients with chronic constipation. Neurogastroenterol Motil 2010;22:991-8.

32. Ke M, Zou D, Yuan Y, et al. Prucalopride in the treatment of chronic constipation in patients from the Asia-Pacific region: a randomized, double-blind, placebo-controlled study. Neurogastroenterol Motil 2012;24:999-e541.

33. Sloots CEJ, Rykx A, Cools M, et al. Efficacy and safety of prucalopride in patients with chronic noncancer pain suffering from opioid-induced constipation. Dig Dis Sci 2010;55:2912-21.

34. Lembo AJ, Schneier HA, Shiff SJ, et al. Two randomized trials of linaclotide for chronic constipation. N Engl J Med 2011;365:527-36.

35. Johnston JM, Kurtz CB, Drossman DA, et al. Pilot study on the effect of linaclotide in patients with chronic constipation. Am J Gastroenterol 2009:104:125-32.

36. Lembo AJ, Kurtz CB, MacDougall JE, et al. Efficacy of linaclotide for patients with chronic constipation. Gastroenterology 2010;138:88695.e1.

37. Johnston JM, Kurtz CB, MacDougall JE, et al. Linaclotide improves abdominal pain and bowel habits in a phase Ilb study of patients with irritable bowel syndrome with constipation. Gastroenterology 2010;139:1877-86.e2.

38. Chey WD, Lembo AJ, Lavins BJ, et al. Linaclotide for irritable bowel syndrome with constipation: a 26-week, randomized, double-blind, placebo-controlled trial to evaluate efficacy and safety. Am J Gastroenterol 2012;107:1702-12. http://www.ncbi.nlm.nih.gov/ pubmed/22986437

39. Rao S, Lembo AJ, Shiff SJ, et al. A 12-week, randomized, controlled trial with a 4-week randomized withdrawal period to evaluate the efficacy and safety of linaclotide in irritable bowel syndrome with constipation. Am J Gastroenterol 2012;107:1714-24; quiz p.1725. http://www.pubmedcentral.nih.gov/articlerender.fcgi?artid= 3504311\&tool=pmcentrez\&rendertype=abstract

40. Atluri DK, Chandar AK, Bharucha AE, et al. Effect of linaclotide in irritable bowel syndrome with constipation (IBS-C): a systematic review and meta-analysis. Neurogastroenterol Motil 2014;26:499509.

41. Shin A, Camilleri M, Kolar G, et al. Systematic review with meta-analysis: highly selective 5-HT4 agonists (prucalopride, velusetrag or naronapride) in chronic constipation. Aliment Pharmacol Ther 2014;39:239-53.

42. Ford AC, Brenner DM, Schoenfeld PS. Efficacy of pharmacological therapies for the treatment of opioid-induced constipation: systematic review and meta-analysis. Am J Gastroenterol 2013;108:1566-74; quiz 1575 .

43. Ford AC, Suares NC. Effect of laxatives and pharmacological therapies in chronic idiopathic constipation: systematic review and meta-analysis. Gut 2011;60:209-18.

44. Videlock EJ, Cheng V, Cremonini F. Effects of linaclotide in patients with irritable bowel syndrome with constipation or chronic constipation: a meta-analysis. Clin Gastroenterol Hepatol 2013;11:1084-92; quiz e68.

45. StatMate [Internet]. (cited 7 Sep 2015). http://www.graphpad.com. laneproxy.stanford.edu/scientific-software/statmate/

46. Chang L, Lembo AJ, Lavins BJ, et al. The impact of abdominal pain on global measures in patients with chronic idiopathic constipation, before and after treatment with linaclotide: a pooled analysis of two randomised, double-blind, placebo-controlled, phase 3 trials. Aliment Pharmacol Ther 2014;40:1302-12.

47. Cuppoletti J, Malinowska DH, Tewari KP, et al. SPI-0211 activates T84 cell chloride transport and recombinant human $\mathrm{CIC}-2$ chloride currents. Am J Physiol Cell Physiol 2004;287:C1173-83.

48. Bryant AP, Busby RW, Bartolini WP, et al. Linaclotide is a potent and selective guanylate cyclase $\mathrm{C}$ agonist that elicits pharmacological effects locally in the gastrointestinal tract. Life Sci 2010;86:760-5.

49. Briejer MR, Prins NH, Schuurkes JA. Effects of the enterokinetic prucalopride (R093877) on colonic motility in fasted dogs. Neurogastroenterol Motil 2001;13:465-72. 